\title{
THEORETICAL AND NUMERICAL STUDY OF WAVE-CURRENT INTERACTION IN STRONGLY-SHEARED FLOWS
}

\author{
Zhifei Dong $^{1}$, James T. Kirby ${ }^{1}$
}

\begin{abstract}
In our study, we establish a new framework to describe the interaction of small amplitude surface gravity waves and strongly sheared currents, where shear can exist in both vertical and horizontal directions. To begin with, we limit the derivation to the case of a narrow-banded slowly varying wave train propagating shoreward in the coastal ocean outside of the surf zone. Accordingly, our problem is assumed to be finite depth without wave breaking. Later we can extend the formulation to describe a spectrum of surface waves and include wave energy dissipation. In contrast to existing formulations, where waves at most feel a weighted depth-average current which follows from a weak-current, weakshear approximation, the present formulation allows for an arbitrary degree of vertical shear, leading to a description of the vertical structure of waves in terms of solutions to the Rayleigh stability equation. Numerical solutions to wave Rayleigh equation are provided based on measured current velocity profile at Columbia River mouth. Wave structure and wave vorticity induced by current vertical shear is discussed.
\end{abstract}

Keywords: wave-current interaction; strong current shear; Rayleigh equation; vortex force

\section{INTRODUCTION}

Wave-current interaction plays a key role in understanding coastal ocean dynamics and near shore sediment transport as well as protecting vessels and coastal structures from severe wave damages. The interaction theory of waves and current has been extensively developed over the past few decades.

The earlier study focuses on the current effects on waves. The simplest and well-known feature of current effects on wave is the Doppler shifting on wave frequency. However, current can never be perfectly uniform in the real world. When current is both horizontally and vertically sheared, it can cause wave refraction, shoaling, breaking, focusing and defocusing, the effect of which is analogous to bathymetry. The refraction theory of waves on current has advanced well with introducing wave action conservation equation. The linear wave theory on vertically sheared weak current is also discussed using both perturbation method and numerical method (Kirby and Chen, 1989). Meanwhile, wave effects on current also draw attention from coastal engineers and oceanographers. In the presence of surface gravity waves, the periodic motion yields a nonzero mean current after time average called Stokes drift. Experimental study also shows that waves can modify current velocity profile by applying wave-averaged forces to mean flow (Kemp and Simons, 1982). Additionally, waves lose energy due to breaking and the lost energy is transferred into current momentum. The wave-averaged effects on current can be interpreted either as radiation stress or as vortex force depending on the treatment of wave advection terms. On the one hand, the concept of radiation stress is adopted to illustrate wave setup/down, the effects of small amplitude surface gravity waves on long waves and tidal current, longshore current and rip current (Longuet-Higgins and Stewart 1960, 1961, 1962, 1964, Longuet-Higgins, 1970 and Bowen, 1969). On the other hand, the alternative of wave-averaged effect vortex force is derived to explain the generation of Langmuir circulation (Garrett, 1976).

With the fast advancement of computational science, three-dimensional numerical modeling of ocean circulation becomes feasible. However, the inclusion of wave-averaged effects in 3D ocean circulation model requires that wave-averaged forces be depth dependent. Efforts have been made to improve the wave effects as depth dependent radiation stress in ocean circulation model (Mellor, 2003, 2008). The depth dependent vortex force formulation is also derived as an alternative to radiation stress (McWilliams et al. 2004). Both of these formulations have been applied in 3D coastal ocean model (Warner et al. 2008 and Uchiyama et al. 2010). However, most of the former studies are based on the assumption of either weak current or strong current with weak vertical shear. Few of these studies consider the interaction of waves with strongly sheared current, in which current vertical shear can affect linear wave dynamics at the leading order.

\section{THEORY}

In our problem, we consider incompressible, inviscid flow and neglect turbulence, which can later be easily added to the governing equation. Thus the motion consists of a steady component and a periodic component. The steady flow is the time-average of total flow, defined as current. It may include tidal current,

\footnotetext{
${ }^{1}$ Center for Applied Coastal Research, University of Delaware, Newark, DE 19716, USA
} 
river current, wave-induced current as well as long waves. The periodic flow is obtained by subtracting the steady flow from total flow that is defined as waves. We consider a narrow-banded small amplitude surface gravity wave train propagating on vertically strongly sheared current. We start with Euler equation and continuity equation, define the coordinates $(x, y, z, t)=(\mathbf{x}, z, t)$ and the motion velocity $\mathbf{u}=(u, v, w)=$ $\mathbf{q}+w \mathbf{i}_{z}$, where $\mathbf{q}=(u, v)$ is horizontal velocity and spacial gradient vector $\nabla=\left(\frac{\partial}{\partial x}, \frac{\partial}{\partial y}, \frac{\partial}{\partial z}\right)=\left(\nabla_{h}, \frac{\partial}{\partial z}\right)$. We use $p$ for pressure, $h$ for water depth and $\eta$ for instantaneous water surface elevation.

$$
\begin{aligned}
\frac{\partial \mathbf{u}}{\partial t}+\mathbf{u} \cdot \nabla \mathbf{u}+\frac{1}{\rho} \nabla p+g \mathbf{i}_{z} & =0 ; \quad-h \leq z \leq \eta \\
\nabla \cdot \mathbf{u} & =0 ; \quad-h \leq z \leq \eta \\
w & =-\mathbf{q} \cdot \nabla_{h} h ; \quad z=-h \\
w & =\frac{\partial \eta}{\partial t}+\mathbf{q} \cdot \nabla_{h} \eta ; \quad z=\eta \\
p & =0 ; \quad z=\eta
\end{aligned}
$$

The curl of momentum equation leads to the vorticity equation, which is used to decribe motion vorticity dynamics.

$$
\frac{\partial \mathbf{\Omega}}{\partial t}+\nabla \times(\boldsymbol{\Omega} \times \mathbf{u})=0 ; \quad-h \leq z \leq \eta
$$

$\boldsymbol{\Omega}=\nabla \times \mathbf{u}=(\xi, \chi)$ is total vorticity. $\xi$ and $\chi$ are horizontal and vertical component, respectively. We consider small amplitude surface gravity waves so that parameter $\epsilon=k a \ll 1$, where $k$ is wave number, $a$ is wave amplitude. The wave motion is described using $\mathrm{e}^{i \Theta}=\cos \Theta+i \sin \Theta$, where $\Theta(\mathbf{x}, t)$ is the phase function. Now we make our problem non-dimensional by introducing a set of characteristic scales. We normalize horizontal distance $x$ and $y$ by characteristic wave length $k_{0}^{-1}$, vertical distance $z$ and surface elevation $\eta$ by water depth $h_{0}$, time $t$ by wave period $\omega_{0}^{-1}$, horizontal velocity $\mathbf{q}$ by wave phase speed $c_{0}=\frac{\omega_{0}}{k_{0}}$, vertical velocity $w$ by $\mu c_{0}$ and $\mu=k_{0} h_{0} \sim O(1)$ for consistency, pressure $p$ by $\rho g h_{0}$, where $\omega_{0}^{2}=g k_{0} \tanh \mu$. In this paper, we focus on finite depth problem. With strong current, strong shear assumption, the current velocity can reach the same magnitude with wave phase speed. The current vertical shear is also comparable to wave frequency. The non-dimensionalized wave variables are of $O(\epsilon)$ for small wave amplitude assumption.

The mean flow is seperated from waves by applying wave-average " $<\cdot\rangle^{\prime \prime}$ over the variable, which is defined either as the spacial average over one wave length or as the temporal average over one wave period.

$$
<\cdot>=\frac{\omega_{0}}{2 \pi} \int_{0}^{2 \pi \omega_{0}^{-1}} \cdot d t=\frac{k_{0}}{2 \pi} \int_{0}^{2 \pi k_{0}^{-1}} \cdot d x
$$

where $\omega_{0}^{-1}$ represents wave period and $k_{0}^{-1}$ represents wave length.

We assume that current variables and wave properties change slowly in time scale and horizontal space scale, which indicates that current and wave amplitude can be treated as constant during one wave period or over one wavelength. We also assume that the ocean bathymetry changes slowly in horizontal space scale. Thus we introduce multiscales in time and space. The fast scales $(\mathbf{x}, z, t)$ are to describe the oscillatory motion and vertical variation while the slow scales $(\mathbf{X}, T)$ are to capture the slowly varying features of both waves and current.

\section{WAVE EQUATION RAYLEIGH EQUATION}

Due to the retention of arbitrarily large shear in the formulation, the leading order problem for wave motion will not be covered by the usual theory for irrotational waves on a depth-uniform flow. Instead, the wave problem is described by a formulation analogous to the Rayleigh stability equation, extended to allow for possible veering of the horizontal component of the current vector with depth. A preliminary description of the resulting system for the spatially uniform case is provided here. We divide the velocity vector $\mathbf{u}=(\mathbf{q}, w)$ into a steady current component $\mathbf{u}^{c}$ and wave component $\mathbf{u}^{w}$, where $\mathbf{u}^{c}(z)=\left(\mathbf{q}^{c}(z), \epsilon w^{c}\right)$ and $\mathbf{q}^{c}$ is horizontal current velocity and $\mathbf{u}^{w}=\epsilon\left(\mathbf{q}^{w}, w^{w}\right)$ is wave orbital velocity. By applying wave average and linearizing boundary conditions, we have wave governing equations are obtained by substracting waveaveraged equations from original equations. 
We seek solution in form of plane periodic waves so let

$$
\begin{aligned}
\mathbf{q}^{w} & =\hat{\mathbf{q}}(z) \mathrm{e}^{i \Theta} \\
w^{w} & =\hat{w}(z) \mathrm{e}^{i \Theta} \\
p^{w} & =\hat{p}(z) \mathrm{e}^{i \Theta} \\
\eta^{w} & =\hat{\eta} \mathrm{e}^{i \Theta} \\
\xi^{w} & =\hat{\xi} \mathrm{e}^{i \Theta} \\
\chi^{w} & =\hat{\chi} \mathrm{e}^{i \Theta}
\end{aligned}
$$

The leading order wave equation leads to a Rayleigh equation describing the vertical structure of $\hat{w}$ together with surface and bottom boundary conditions. $\sigma(z)$ is intrinsic frequency which is the wave frequency observed by the current.

$$
\begin{array}{rlrl}
\sigma \frac{\partial^{2} \hat{w}}{\partial z^{2}}-\left(\sigma k^{2}+\frac{\partial^{2} \sigma}{\partial z^{2}}\right) \hat{w} & =0 ; & & -h \leq z \leq 0 \\
\hat{w} & =0 ; & z=-h \\
\sigma_{s}^{2} \frac{\partial \hat{w}}{\partial z}-\left(\sigma_{s} \frac{\partial \sigma}{\partial z}+g k^{2}\right) \hat{w} & =0 ; \quad z=0 \\
\sigma(z) & =\omega-\mathbf{k} \cdot \mathbf{q}^{c}(z)
\end{array}
$$

\section{NUMERICAL SOLUTION TO RAYLEIGH EQUATION}

In the irrotational wave theory, the wave velocity has the form

$$
\begin{aligned}
& \hat{w}(z)=-i \omega \hat{\eta} \frac{\sinh k(h+z)}{\sinh k h} \\
& \hat{\mathbf{q}}(z)=\frac{\omega \hat{\eta} \mathbf{k}}{k} \frac{\cosh k(h+z)}{\sinh k h}
\end{aligned}
$$

For wave Rayleigh equation, we can also assume a non-dimensional depth dependent function $f(z)$. It is noteworthy that slow scales variation does not involve in the leading order Rayleigh equation. We set slow scales aside and treat wave variables as function of only fast scales.

$$
\begin{aligned}
& \hat{w}(z)=-i \sigma_{s} \hat{\eta} f(z) \\
& \hat{p}(z)=-\frac{\rho \hat{\eta} \sigma_{s}^{2}}{k^{2}}\left(f \frac{d L}{d z}-L \frac{d f}{d z}\right) \\
& \hat{\mathbf{q}}(z)=-\frac{\hat{\eta} f}{L} \frac{d \mathbf{q}^{c}}{d z}+\frac{\sigma_{s} \hat{\eta} \mathbf{k}}{k^{2}}\left(\frac{d f}{d z}-\frac{f}{L} \frac{d L}{d z}\right)
\end{aligned}
$$

$\sigma_{s}$ is surface intrinsic frequency. We also introduce the vertical structure function of intrinsic frequency.

$$
\begin{aligned}
\sigma(z) & =\sigma_{s} L(z) \\
f^{\prime} & =\frac{d f}{d z}
\end{aligned}
$$

The Rayleigh equation can be rewritten as followinig

$$
\begin{aligned}
f^{\prime \prime}-\left(k^{2}+\frac{L^{\prime \prime}}{L}\right) f & =0 ; \quad-h \leq z \leq \eta^{c} \\
f(-h) & =0 ; \\
f^{\prime} & =\left(L^{\prime}+\frac{g k^{2}}{\sigma_{s}}\right) f ; \quad z=\eta^{c}
\end{aligned}
$$

For the strong current with arbitrary shear, Rayleigh equation has no analytical solution. Following Kirby and Chen (1989), two methods can be used to solve Rayleigh equation: direct numerical solving and perturbation approximation. An exact solution to Rayleigh equation can be obtained by transforming the boundary value problem into an initial value problem and solve it using shooting method. First we switch 
the coordinate from $-h \leq z \leq \eta^{c}$ to $-H \leq z \leq 0$, where $H=h+\eta^{c}$ and introduce a new variable $Q(z)=\frac{f}{H f^{\prime}}$ and non-dimensionalized vertical coordinate $\hat{z}=\frac{z}{H}$ and get them into Rayleigh equation, we have

$$
\begin{aligned}
\frac{d Q}{d \hat{z}} & =1-\gamma^{2} Q^{2} ; \quad-1 \leq \hat{z} \leq 0 \\
\gamma^{2}(\hat{z}) & =(k H)^{2}+\frac{L^{\prime \prime}}{L} \\
Q & =\frac{\sigma_{s}^{2}}{g k^{2} H+\sigma_{s}^{2} L^{\prime}} ; \quad \hat{z}=0 \\
Q & =0 ; \quad \hat{z}=-1
\end{aligned}
$$

Now look at the relation between $L(\hat{z})$ and current profile. We also assume that current horizontal velocity $\mathbf{q}^{c}(\hat{z})=q_{s}^{c} \mathbf{I}(\hat{z}) . q_{s}^{c}$ is the surface current velocity magnitude and the nondimensional vector function $\mathbf{I}(\hat{z})$ represents current direction and vertical profile. It is noteworthy that the current direction may rotate over depth. The intrinsic frequency is expressed using current.

$$
\sigma(\hat{z})=\omega-q_{s}^{c} \mathbf{k} \cdot \mathbf{I}(\hat{z})=\sigma_{s} L(\hat{z})
$$

So that

$$
L(\hat{z})=\frac{\omega}{\sigma_{s}}-\frac{q_{s}^{c} \mathbf{k} \cdot \mathbf{I}(\hat{z})}{\sigma_{s}}
$$

Solving the problem requires that current velocity $\mathbf{q}^{c}(z)$ distribution be regarded as known. So it is treated as a set of known data points in the water column and approximated using polynomial expansion.

$$
\begin{aligned}
I(z) & =\frac{\mathbf{q}^{c}}{q_{s}^{c}}+\mathbf{A}_{1} \hat{z}+\mathbf{A}_{2} \hat{z}^{2}+\mathbf{A}_{3} \hat{z}^{3}+\ldots \\
L(z) & =1+B_{1} \hat{z}+B_{2} \hat{z}^{2}+B_{3} \hat{z}^{3}+\ldots \\
B_{n} & =-\frac{q_{s}^{c} \mathbf{k} \cdot \mathbf{A}_{\mathbf{n}}}{\sigma_{s}} \quad(n=1,2,3 \ldots)
\end{aligned}
$$

Then we can get numerical solutions for both $Q(\hat{z})$ and phase speed $c$ using shooting method. Specifically, we firstly give a starting value for $c$, then march the solution from bottom $\hat{z}=-1$ to the surface $\hat{z}=0$ to get $Q(0)$. At the same time, we can get the value of $Q(0)$ directly through surface boundary condtion. If the difference of these two $Q(0)$ is within a tolerancible range, we find the solution for $Q(z)$ and $c$. Otherwise, we use the new $c$ from surface boundary condition and march the solution again until we are satisfied with the magnitude of error.

\section{APPLICATION: COLUMBIA RIVER}

We use the measured current profile at Columbia river mouth to illustrate current vertical shear effects on wave structures. The dataset are released as part of RISE (River Influences on Shelf Ecosystems) project oberved by Ocean Mixing Group, Oregon State University (Kilcher and Nash, 2010). Several cruises have been taken at the Columbia River mouth in July 2004 . We choose the data at location $46.24^{\circ} \mathrm{N}, 124.17^{\circ} \mathrm{W}$ taken during an ebb at the river mouth. The current velocity is mainly eastward. Therefore, we may reduce our problem to a $2 \mathrm{DV}$ case without considering current direction rotation over depth. The wave vorticity balance equation gives the expression of wave vorticity generated by current vertical shear.

$$
\begin{aligned}
& \hat{\xi}=-i\left[\frac{\partial \mathbf{q}^{c}}{\partial z} \frac{\left(\xi^{c} \cdot \mathbf{k}\right) \hat{w}}{\sigma^{2}}+\frac{\partial \xi^{c}}{\partial z} \frac{\hat{w}}{\sigma}\right]-\frac{\left(\xi^{c} \cdot \mathbf{k}\right) \hat{\mathbf{q}}}{\sigma} \\
& \hat{\chi}=-\frac{\left(\xi^{c} \cdot \mathbf{k}\right) \hat{w}}{\sigma}
\end{aligned}
$$

Where $\xi^{c}$ is current horizontal vorticity.

$$
\xi^{\mathbf{c}}=\mathbf{i}_{\mathbf{z}} \times \frac{\partial \mathbf{q}^{\mathbf{c}}}{\partial \mathbf{z}}
$$

We consider a group of waves with peak frequency at $12 \mathrm{~s}$ traveling westward against the river discharge. The result shows how the opposing current can affect wave velocity structure. Figure 1 gives 


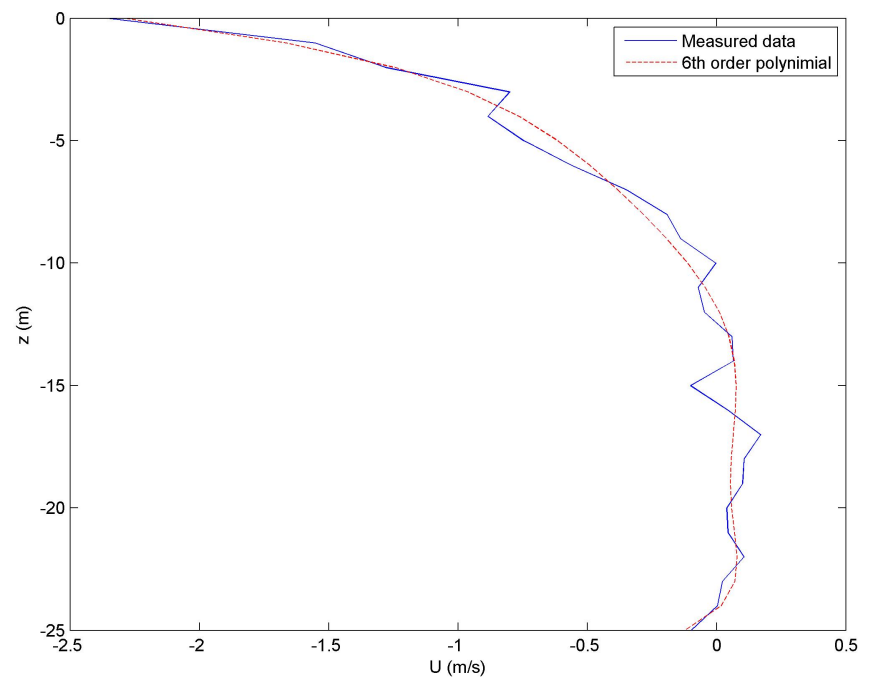

Figure 1. Current profile at Columbia river mouth

(Solid line: measured data, Dashed line: 6th order polynomial fitting)

current velocity profile at Columbia river mouth. The 6th order polynomial expansion is used to fit the current profile. During the ebb from high tide to low tide, the river flow is offshore. A strong vertical shear is formed in the upper layer in the figure. It is well known that the motion of surface gravity wave is confined in the upper layer as well as wave-averaged force on the mean flow. Hence, the strong current shear near the surface can modify the wave structure and wave averaged force significantly. The wave vortex force formulation derived in our study indicates that the wave vorticity dynamics, besides Stokes drift and current shear coupled terms, is involved in vortex force. It is necessary to quantify wave vorticity on the strongly sheared current. Figure 2 gives the orbital velocity of pure waves and waves on the opposing current shown in Figure 1 during one wave period. In the presence of an opposing current, wave orbital velocity is strengthened through intrinsic frequency. The horizontal orbital velocity is much larger than current-free case, while the vertical velocity is slightly changed by the current. The current shear effects on wave structure can also be illustrated through wave vorticity distribution in Figure 3. Strong current shear near water surface generates wave voriticity confined near the surface. The evolution of wave vorticity coupled with wave orbital velocity will force the mean flow in return.

\section{CONCLUSIONS AND FUTURE WORK}

In this paper, we formulate the interaction of a narrow-banded small amplitude surface gravity wave train with strongly sheared current in vertical direction. The first order wave equation leads to the Rayleigh stability equation. The expression of wave vorticity induced by current vertical shear is given in this problem. The wave Rayleigh equation is transformed into an initial value problem and solved numerically using the shooting method. As an application, current velocity profile data at Columbia river mouth is used to illustrate current shear effects on the wave structure. The 6th order polynomial expansion is applied to fit the measured current profile. We suppose a group of waves with peak frequency at $12 \mathrm{~s}$ traveling shoreward on an opposing river current. Due to strong current and vertical shear, wave orbital velocity is strengthened and wave vorticity is generated. The resulting formulation that leads to a conservation law for wave action and forcing terms for the description of mean flow using the Craik-Leibovich vortex force formulation will be discussed in another paper. This new framework of wave-current interaction can be applied to numerical model based on ROMS/SWAN to study dynamics in coastal waters.

\section{ACKNOWLEDGMENTS}

This work is sponsored by the National Science Foundation, Physical Oceanography Program. 


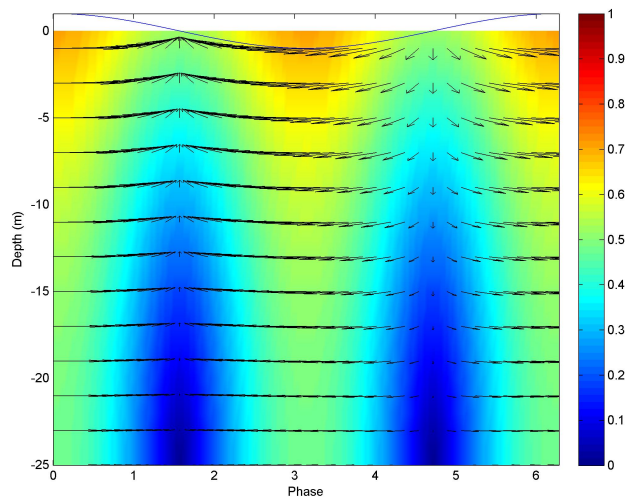

(a)

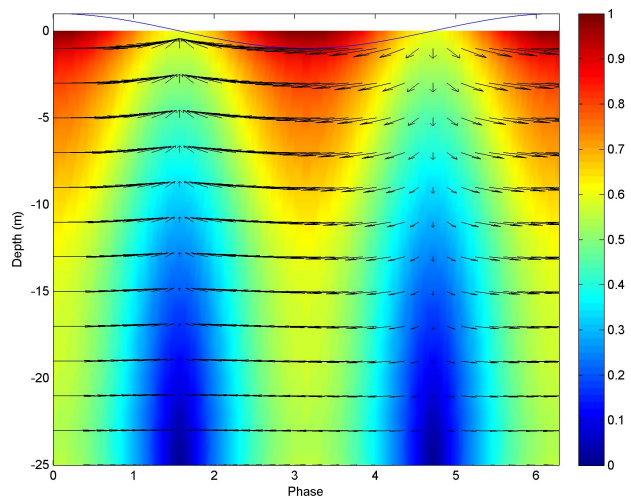

(b)

Figure 2. Wave orbital velocity during one wave period (Solid line indicating wave crest (a) Pure waves (b) Waves on opposing current)

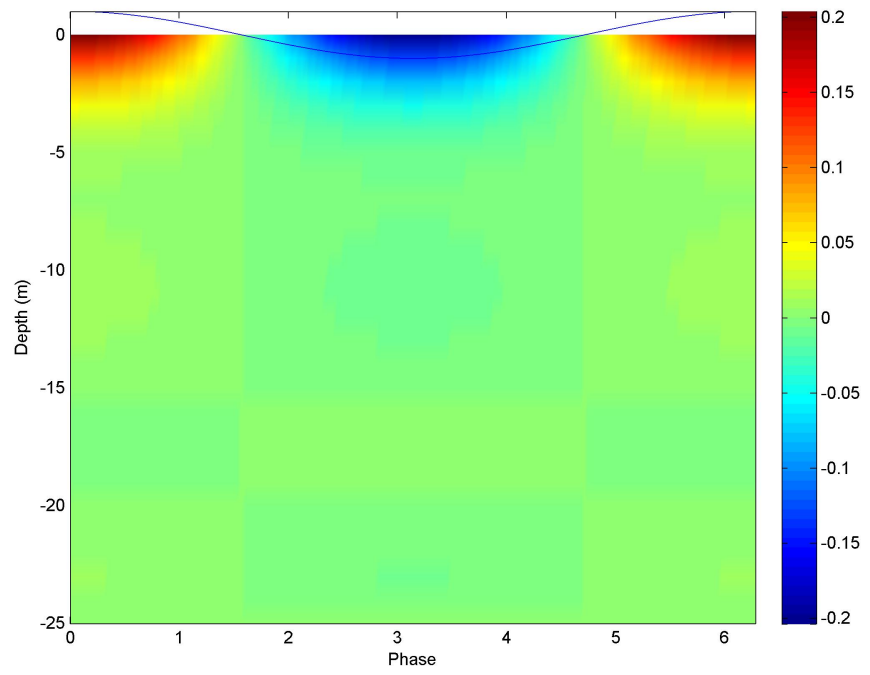

Figure 3. Wave vorticity induced by current vertical shear 


\section{REFERENCES}

Garrett, C., 1976, “Generation of Langmuir circulations by surface waves-A feedback Mechanism, J. Mar. Res., 34, 116-130.

Kemp, P. H. \& Simons, R. R., 1982, "The interaction between waves and a turbulent current: waves propagating with the current", J. Fluid Mech., 116, 227-250.

Kirby, J. T. \& Chen, T-M., 1989, "Surface waves on vertically sheared flows: approximate dispersion relations", J. Geophys. Res., 94, 1013-1027.

Longuet-Higgins, M. S. \& Stewart, R. W., 1960, "Changes in the form of short gravity waves on long waves and tidal currents". J. Fluid Mech., 8, 565-583.

Longuet-Higgins, M. S. \& Stewart, R. W., 1961, "The changes in amplitudeof short gravity waves on steady non-uniform currents", J. Fluid Mech., 10, 529-549.

Longuet-Higgins, M. S. \& Stewart, R. W., 1962, "Radiation stress and mass transport in gravity waves, with applications to 'surf-beats'", J. Fluid Mech., 13, 481-504.

Longuet-Higgins, M. S. \& Stewart, R. W., 1964, "Radiation stress in water waves: a physical discussion, with applications", Deep-Sea Res. Oceanogr. Abstr., 11, 529-562.

Longuet-Higgins, M. S., 1970, "Longshore currents generated by obliquely incident sea waves I.", $J$. Geophys. Res., 75, 6778-6789.

McWilliams, J. C., Restrepo, J. M. and Lane, E. M., 2004, “An asymptotic theory for the interaction of waves and currents in coastal waters", J. Fluid Mech., 511, 135-178.

Mellor, G., 2003, "The three-dimensional current and surface wave equations", J. Phys. Oceanogr., 33, 1978-1989.

Mellor G., 2008, "The depth dependent current and wave interaction equations: a revision", J. Phys. Oceanogr., 38, 2587-2596.

Uchiyama, Y., McWlliams, J. C., Shchepetkin, A. F., 2010, "Wave-current interaction in an oceanic circulation model with a vortex-force formalism: application to the surf zone", Ocean Modeling, 34, 16-35.

Warner, J. C., Sherwood, C. R., Signell, R. P., Harris, C. K., Arango, H. G., 2008, "Development of a three-dimensional, regional, coupled wave, current, and sediment-transport model", Computers $\mathcal{E}$ Geosciences, 34, 1284-1306. 